
\title{
$\angle S$ Research Suare \\ Feasibility of diffusion kurtosis imaging in evaluating cervical spinal cord injury in multiple sclerosis
}

BingYang Bian

First Hospital of Jilin University

BoXu Zhou

First Hospital of Jilin University

\section{ZhiQing Shao}

First Hospital of Jilin University

XiaoNa Zhu

First Hospital of Jilin University

YiGe Jie

First Hospital of Jilin University

Dan Li ( $\sim$ li_d@jlu.edu.cn )

First Hospital of Jilin University

\section{Research Article}

Keywords: multiple sclerosis, cervical spinal cord, magnetic resonance imaging, diffusion kurtosis imaging, extended disability scale score

Posted Date: December 9th, 2022

DOI: https://doi.org/10.21203/rs.3.rs-1114527/v2

License: (c) (i) This work is licensed under a Creative Commons Attribution 4.0 International License. Read Full License 


\section{Abstract \\ Background and purpose}

At present, it is a difficult problem to evaluate cervical spinal cord (CSC) injury in multiple sclerosis (MS). To assess gray matter (GM), white matter (WM), lesions of MS and the thrapeutic effect using diffusion kurtosis imaging (DKI).

\section{Material and Methods}

A total of 78 subjects (48 of MS and 30 of health ) perform routine MR scan and DKI of CSC. DKI-metrics were measured in the lesions, normal-appearing GM and WM. The MS patients were divided into two groups according to the presence or absence of T2 hyperintensity. The disability of the two groups was assessed by the extended disability status scale (EDSS) before and after treatment.

\section{Results}

1) Significant differences were detected in DKI metrics between MS and healthy $(P<0.05)$ and between patients with CSC T2-hyperintense and without T2-hyperintense $(P<0.001) ; 2)$ Compared to healthy, GMMK, GM-RK and WM-FA, WM-AD were statistically reduced in patients without T2-hyperintense $(P<0.05)$. 3) Significant differences were observed in DKI metrics between patients with T2-hyperintense after therapy $(P<0.05)$, as well as GM-MK and WM-FA, WM-AD in patients without T2-hyperintense $(P<0.05)$; 4) EDSS was correlated with MK values, as well as EDSS scores and MK values after therapy.

\section{Conclusions}

1) DKI-metrics can detect and quantitatively evaluate the changes in cervical spinal cord micropathological structure; 2) MK and RK values are sensitive metrics to detect the damage of gray matter; 3) MK values quantitatively evaluate the clinical disability progression and the therapeutic effect in MS patients.

\section{Introduction}

Multiple sclerosis (MS) is the most common neuroimmunological demyelinating disease and the most common non-traumatic disability in young adults[1,2]. The cervical spinal cord (CSC) is a vulnerable region. Extensive abnormalities in CSC are correlated with neurological deficits and the development of clinical disability in MS patients[3, 4]. However, there is a clinicoradiological contradiction that clinical disability and routine MR parameters, including the number and volume of T2-hyperintense CSC lesions, show poor correlation and the so-called normal-appearing of CSC in MRI[5-11]. Fortunately, new quantitative imaging, such as diffusion tensor imaging (DTI)[12] and diffusional kurtosis imaging (DKI) 
[13], assessed microstructural CSC abnormalities in MS patients, which could surmount this paradox and provide novel biomarkers to monitor disease progression. DTI is based on the assumption that diffusing water molecules follow a Gaussian distribution and is a significant MRI used to observe the displacement of water molecules at a microscopic level[13]. Spinal cord DTI (typical b-value $=0,1000,1500 \mathrm{~s} / \mathrm{mm}^{2}$ ) has now been used in MS research for almost a decade[14]. DTI metrics include fractional anisotropy (FA), mean diffusivity (MD), axial diffusivity(AD) and radial diffusivity (RD). FA, an index of diffusion anisotropy and integrity, could be biomarkers of WM damage[12]. MD, AD and RD are diffusion metrics calculated along the averaged eigenvectors, the principal and the perpendicular, respectively[15]. Typically, the displacement of water molecules is restricted by barriers, such as cell membranes and organelles. Hence, DTI of CSC may be inappropriate in the biological structures. In addition, DTI modeled on single fiber orientation in each voxel has limited reliability for crossing fibers and provides less valuable information in tissues such as GM because the microstructure of GM is characterized as isotropic. Although several correlations have been established between quantitative MR metrics of CSC and clinical disability in MS, the gray matter of CSC is rarely observed[16, 17]. DKI (typical b-value $=0$, $500,1000,1500,2000,2500 \mathrm{~s} / \mathrm{mm}^{2}$ ) is an advanced form of diffusion analysis, which enables the quantification of non-Gaussian diffusion through the estimation of the diffusional kurtosis[12]. Thus, DKI may accurately reflect subtle micropathological abnormalities in GM in vivo compared to DTI[18]. DKI metrics include mean kurtosis (MK), axial kurtosis (AK) and radial kurtosis (RK). MK, a index of tissue microstructural complexity, has been shown to be sensitive to microstructural pathological changes in both anisotropic and isotropic tissue compared to FA and MD. AK are kurtosis in the direction of the principal diffusion tensor eigenvector. RK are kurtosis averaged over all diffusion directions perpendicular to the principal diffusion tensor eigenvector[15].

As the Expanded Disability Status Scale (EDSS)-the clinical scale most frequently used to quantify disability in MS-is heavily weighted toward motor disability. The measurement of spinal cord damage provides clinically relevant information than the assessments of brain damage.

Through DTI of cervical spinal cord, Oh $\mathrm{J}$ found that the FA, MD values were more abnormal in the highvs low-disability subgroup of MS patients[11]. One study showed that the DTI metrics in AD and RD show a pathological increase, and FA shows a pathological decrease in cervical spinal cord T2-hyperintense lesions[14]. Through DKI of cervical spinal cord in patients with MS, Raz E found that cervical cord white matter FA and gray matter MK value were significantly decreased compared with controls[12]. The present study aimed to: 1) quantitatively evaluate microstructural damage of lesion and normalappearing GM and WM in the CSC of MS patients compared to healthy subjects using MK, MD, and FA value; 2) determine whether MK provides additional information about GM abnormalities compared to MD and FA values; 3 ) assess the association between DKI metrics of CSC and clinical disability assessed by EDSS score before and after therapy.

\section{Material And Methods}




\section{Study population}

From January 2018 to October 2019, 48 consecutive patients with MS diagnosed by 2017 McDonald criteria[19] in our institution were subjected to cervical spinal cord MRI before and after therapy. The exclusion criteria were as follows: 1 ) other related diseases, such as cervical trauma and neuromyelitis optica; 2) contraindications to performing MRI; 3) steroid treatment in the 3 months before MRI. After MS patients had undergone a disease modifying therapies (DMTs)[19], MR scans were performed again when no corticosteroids treatment during the last month. The following demographic and clinical variables were acquired for each patient: age, sex, disease duration, and degree of disability assessed by EDSS score. An experienced neurologist recorded the EDSS score before and after one course of treatment. According to CSC T2-hyperintense, MS patients were divided into patients with T2hyperintense lesions and patients with no T2-hyperintense lesions. Moreover, 30 healthy sex- and agematched adults comprised the control group.

\section{MRI acquisition}

All images were acquired by a 3.0T MR scanner (Philips Ingenia; Philips Medical Systems, Best, The Netherlands) using a 16-channel head and neck coil. The examinee was placed in the supine position and informed not to move the body as much as possible, breathe calmly, and avoid swallowing movements and cough to reduce motion artifacts. For each subject, cervical spinal cord MRI protocol was as follows:

1. Sagittal T1-weighted, by using a spin-echo MR imaging sequence, 15 contiguous sections was acquired $(T R / T E=550 / 6.4 \mathrm{~ms}$; echo-train length $=4$; section thickness $/ \mathrm{gap}=3 / 0 \mathrm{~mm} ; \mathrm{FOV}=240 \mathrm{~mm}$; matrix $=256 \times 256$ ).

2. Sagittal T2-weighted, by using a fast spin-echo MR imaging sequence, 15 contiguous sections were acquired and used to plan axial imaging such that the axial slice stack was centered on the C4-C5 vertebral disc and perpendicular to the spinal cord $(T R / T E=3263 / 95 \mathrm{~ms}$; echo-train length $=36$; section thickness $/ \mathrm{gap}=3 / 0 \mathrm{~mm}$; FOV $=240 \mathrm{~mm}$; matrix $=256 \times 256$ ).

3. Axial T2*-weighted, centered on the $\mathrm{C} 4-\mathrm{C} 5$ vertebral disc, were acquired using an averaged multiple fast-field echo (mFFE) sequence with 5 echoes and 35 contiguous axial sections was acquired $\left(\mathrm{TR} / \mathrm{TE}=700 / 7.20 \mathrm{~ms}, 4\right.$ additional echoes where $\Delta \mathrm{TE}=8.83 \mathrm{~ms}$; flip angle $=28^{\circ}$; section thickness $/$ gap $=3 / 0 \mathrm{~mm} ; \mathrm{FOV}=150 \times 150 \mathrm{~mm}^{2} ;$ matrix $=512 \times 512$ ). The mFFE sequence provided high-resolution detail of the cervical spinal cord, including GM, WM, and was used to delineate structures of regions of interest[20].

4. Axial DKI sequence, the center position is the same as axial T2-weighted, using a twice-refocused spin-echo diffusion sequence with an echo-planar imaging readout with 32 different diffusionencoding directions and 35 contiguous axial sections. For each direction, 4 b-values $(0,700,1400$, and $2100 \mathrm{~s} / \mathrm{mm}^{2}$ ) were used (TR/TE $=3100 / 101 \mathrm{~ms}$; section thickness $/ \mathrm{gap}=3 / 0 \mathrm{~mm} ; \mathrm{FOV}=80 \times 80$ $\mathrm{mm}^{2} ;$ matrix $\left.=512 \times 512\right)$. The gradient length $(\delta)$ and time between the two leading edges of the diffusion gradients $(\Delta)$ were 9.8 and $44.1 \mathrm{~ms}$, respectively. F.O.S. is on, one RF pulse is collected 
within FOV, and the other RF pulse is collected in the area of 50\% FOV on both sides of the phase coding direction.

\section{Image analysis}

All DKI data were imported in the in-house software (Diffusion Kurtosis Estimator) in MATLAB to spatial smoothing, correct for eddy distortions, median filtering, denoising and correction for Gibbs ringing artifacts, which has been validated and reported before[12, 15]. We use the two-dimensional (2D) rigidbody algorithm reported in the literature to coregistering all diffusion weighted image to T2*-weighted image and obtain FA, MD and MK maps of DKI-derived indices[12, 21]. DKI metrics included FA, MD, and MK values. First, the FA and MD values were calculated in the DTI model. Second, the diffusion kurtosis was calculated on a voxel-by-voxel basis as described previously [18]. Four $b$ values were used in the following equation:

$\ln [S(b)]=\ln [S(0)]-b \star D_{a p p}+1 / 6 * b^{2} * D^{2}{ }_{a p p} * K_{a p p}$

where $\mathrm{D}_{\mathrm{app}}$ is the apparent diffusion coefficient for the given direction, and $\mathrm{K}_{\mathrm{app}}$ is the apparent kurtosis coefficient that is dimensionless. Then, region of interest (ROI) was used to measure the DK metrics of the spinal cord between $\mathrm{C} 1$ and $\mathrm{C} 7$ levels, generating 35 values for each of the DKI metrics in each subject (1680 spinal cord sections for 48 patients and 1050 spinal cord section for 30 controls). Of the 2730 sections, 205 included T2*-weighted visible lesions, whereas the remaining were classified as normal-appearing CSC. The lesions were outlined on the T2*-weighted images using the MIPAV (Medical Image Processing, Analysis, and Visualization) application for quantitative analysis and visualization of medical images (http://mipav.cit.nih.gov). Then, the outlined ROls were automatically transferred onto the corresponding DKI maps by the dTV II FZR softwareto obtain quantitative values from each parameter[22]. In addition, normal-appearing GM and WM from patients and healthy controls were measured using rectangular ROIs (size: 2 voxels, $3.0 \mathrm{~mm}^{2}$ ) placed on the $T 2^{*}$-weighted images in the right and left anterior gray horns and the dorsal column (reduce artifacts caused by the flow of the cerebrospinal fluid, the data within the WM had to be measured as close to the inner side of the spinal cord as possible) in 35 contiguous sections at the $\mathrm{C} 1-\mathrm{C} 7$ level, as described in Fig. 1. Then, the dTV II FZR software allowed for copying of the ROls and guaranteed the evaluation of the same region with corresponding DKI maps. The normal-appearing GM/WM metrics were calculated by averaging the results obtained in all ROIs (70 ROIs for GM and 35 ROls for WM). Thus, each subject contributed one value for each of MK, MD, and FA for the total spinal cord, including lesions and normal-appearing GM/WM. ALL image data processing and evaluation were performed by a neuroradiologist who specialized in neuroimmunology. Sagittal T2-weighted and axial T2*-weighted were used to identify and count the lesions in the CSC by a neuroradiologist who worked for 15 years.

\section{Statistical Analysis}


SPSS software (version 21.0; SPSS, Chicago, IL, USA) was used for statistical analysis. Intra-class correlation coefficients were used to evaluate the agreement between the two readers for each DKI parameters. All demographic, clinical, and EDSS scores were examined with the non-parametric Wilcoxon rank-sum test or the Kruskal-Wallis test because the values did not follow normal distributions. An ANOVA model, adjusted for age and sex, was used to assess the differences in DKI metrics among the study groups. A $\chi^{2}$ test was used for patient sex comparison. Before and after therapy, the DKI metrics in the MS group were compared by paired-sample t-test. The correlations between EDSS score and DKI metrics from the lesions and the GM and WM were determined using Pearson's correlation coefficient. $P<0.05$ indicated statistical significance.

\section{Results}

\section{Demographics}

Subject demographics and clinical data for our patient and control groups are provided in Table 1. No significant differences were detected in sex $(P=0.632)$ among the three groups. Among 48 patients with MS, 31 had relapsing-remitting (RR) MS, 10 had secondary progressive (SP) MS, and 7 presented primaryprogressive (PP) MS. A total of 25 T2 hyperintense lesions were identified in 15/ 48 (31\%) patients with MS (10 with SPMS, 4 with PPMS, and 1 with RRMS). A median of 2 (range: 1-3) lesions was noted in each patient. One cervical spinal cord T2 hyperintense lesion was detected in 8/48 (17\%) patients with MS (5 with SPMS, 2 with PPMS, and 1 with RRMS), two cord lesions were detected in 4/48 (8\%) patients (2 with SPMS and 2 with PPMS), and three cord lesions were detected in 3/48 (6\%) patients (2 with SPMS and 1 with PPMS). T2-weighted images, appearing normal, were found in the other $33 / 48(69 \%)$ patients ( 30 with RRMS, 2 with PPMS, and 1 with SPMS). The MS patients with T2 hyperintense lesions were older, had prolonged disease duration and had higher EDSS scores than the patients with no T2 hyperintense (all $P<0.001$ ). 
Table 1

Main demographic of clinical data of the study groups

\begin{tabular}{|c|c|c|c|c|}
\hline Parameter & $\begin{array}{l}\text { Healthy } \\
\text { Subjects ( } n= \\
30)\end{array}$ & $\begin{array}{l}\text { Patients with T2 } \\
\text { hyperintense }(n=15)\end{array}$ & $\begin{array}{l}\text { Patients with no T2 } \\
\text { hyperintense }(n=33)\end{array}$ & $P$ value \\
\hline Sex & & & & $.632^{*}$ \\
\hline Men & 11 & 7 & 12 & \\
\hline Women & 19 & 8 & 21 & \\
\hline Mean age (y) & $44.2(19-55)$ & $45.7(21-56)$ & $39.6(17-44)$ & $.001^{\dagger}$ \\
\hline $\begin{array}{l}\text { Median no. of CSC T2 } \\
\text { hyperintense lesions }\end{array}$ & - & $2(1-3)$ & - & \\
\hline $\begin{array}{l}\text { Median disease } \\
\text { duration (y) }\end{array}$ & - & $21(2-44)$ & $12(0.25-41)$ & $.001^{t+}$ \\
\hline Median EDSS score & - & $6.0(1.5-8.0)$ & $3.0(1.0-6.0)$ & $.001^{++}$ \\
\hline \multicolumn{4}{|c|}{ Note.-Data in parentheses are range. Abbreviation: EDSS = Expanded Disability Status Scale. } & \\
\hline \multicolumn{5}{|c|}{${ }^{\dagger}$ Determined with AVNOA. } \\
\hline \multicolumn{5}{|c|}{${ }^{\dagger+}$ Determined with Kruskal-Wallis test. } \\
\hline
\end{tabular}

\section{Comparison of DKI metrics between healthy subjects and MS patients}

Inter-rater agreement for DKI metrics between the two readers was found to be good (range 0.8 to 0.9 ). Figure 2 shows the distribution of DKI metrics in CSC of MS patients and healthy subjects. Compared to healthy subjects $(n=30), \mathrm{FA}, \mathrm{AD}, \mathrm{MK}$, RK were significantly decreased (all $P<0.05)$ and MD, RD, AK value were increased $(P<0.05)$ in MS patients $(n=48)$.

Table 2 shows that DKI metrics of GM placed the ROIs in bilateral ventral horns and WM placed the ROIs in the dorsal column, which were compared at the C1-C7 level in healthy subjects $(n=30)$ and MS patients with no T2 hyperintense lesions at that level $(n=33)$. In MS patients, normal-appearing GM-MK, GM-RK and WM-FA, WM-AD values were significantly decreased compared to those in healthy subjects ( $P$ $=0.018,<0.001$ and $<0.001,0.0211$ respectively), while other DKI parameters were not significantly different across the two groups of subjects. 
Table 2

Comparison of DKI metrics between MS patients with no T2 hyperintense and healthy subjects

\begin{tabular}{|c|c|c|c|c|c|}
\hline Metrics§ & Location & $\begin{array}{l}\text { Patients with no T2 } \\
\text { hyperintense }(n=33)\end{array}$ & $\begin{array}{l}\text { Healthy subjects } \\
(n=30)\end{array}$ & Parameter & $\begin{array}{l}P \\
\text { value }^{+}\end{array}$ \\
\hline \multirow[t]{2}{*}{ MK } & GM & $1.08 \pm 0.02$ & $1.15 \pm 0.04$ & $t=-9.807$ & .018 \\
\hline & WM & $1.12 \pm 0.13$ & $1.11 \pm 0.03$ & $t=-1.789$ & .079 \\
\hline \multirow[t]{2}{*}{ MD } & GM & $0.71 \pm 0.01$ & $0.70 \pm 0.02$ & $t=0.899$ & .372 \\
\hline & WM & $0.90 \pm 0.02$ & $0.89 \pm 0.07$ & $t=-1.059$ & .294 \\
\hline \multirow[t]{2}{*}{ FA } & GM & $0.80 \pm 0.12$ & $0.81 \pm 0.07$ & $t=-1.828$ & .073 \\
\hline & WM & $0.52 \pm 0.04$ & $0.63 \pm 0.03$ & $t=-17.749$ & $<.001$ \\
\hline \multirow[t]{2}{*}{$A D$} & GM & $1.54 \pm 0.02$ & $1.55 \pm 0.01$ & $t=-1.021$ & .284 \\
\hline & WM & $1.67 \pm 0.03$ & $1.78 \pm 0.04$ & $t=-8.904$ & .0211 \\
\hline \multirow[t]{2}{*}{ RD } & GM & $0.65 \pm 0.04$ & $0.64 \pm 0.05$ & $t=-2.104$ & .081 \\
\hline & WM & $0.68 \pm 0.02$ & $0.67 \pm 0.02$ & $t=-1.504$ & 0.231 \\
\hline \multirow[t]{2}{*}{ AK } & GM & $0.59 \pm 0.01$ & $0.58 \pm 0.02$ & $t=0.922$ & 0.512 \\
\hline & WM & $0.60 \pm 0.02$ & $0.59 \pm 0.03$ & $t=0.899$ & 0.458 \\
\hline \multirow[t]{2}{*}{ RK } & GM & $0.91 \pm 0.03$ & $1.27 \pm 0.02$ & $t=-16.904$ & $<.001$ \\
\hline & WM & $0.94 \pm 0.01$ & $0.95 \pm 0.02$ & $t=-0.904$ & 0.436 \\
\hline \multicolumn{6}{|c|}{$\begin{array}{l}\text { Note.-Abbreviation: } \mathrm{MK}=\text { mean kurtosis (unitless); } \mathrm{MD}=\text { mean diffusivity }\left(\times 10^{-3} \mathrm{~mm}^{2} / \mathrm{s}\right) ; \mathrm{FA}= \\
\text { fractional anisotropy (unitless); } \mathrm{AD}=\text { axial diffusivity }\left(\times 10^{-3} \mathrm{~mm}^{2} / \mathrm{s}\right) ; \mathrm{RD}=\text { radial diffusivity }\left(\times 10^{-3}\right. \\
\left.\mathrm{mm}^{2} / \mathrm{s}\right) ; \mathrm{AK}=\text { axial kurtosis (unitless); } \mathrm{RK}=\text { radial kurtosis (unitless). } \mathrm{GM}=\text { gray matter; } \mathrm{WM}=\text { white } \\
\text { matter. DKI metrics from GM and WM for patients with no T2 hyperintense and healthy subjects. }\end{array}$} \\
\hline
\end{tabular}

Table 3 shows that DKI parameters of sections from lesions were compared to the values of sections from normal-appearing CSC in MS patients with T2 hyperintense $(n=15)$ and MS patients with no T2 hyperintense lesions $(n=33)$. The six metrics differed significantly: MK, FA, AD, RD, AK and RK values were lower and MD values were higher in lesions than in normal-appearing CSC (all $P<0.001)$. 
Table 3

Comparison of DKI metrics between MS patients

\begin{tabular}{|c|c|c|c|c|}
\hline Metrics§ & $\begin{array}{l}\text { Patients with T2 hyperintense } \\
(n=15)\end{array}$ & $\begin{array}{l}\text { Patients with no T2 hyperintense } \\
(n=33)\end{array}$ & Parameter & $\begin{array}{l}P \\
\text { value }^{\dagger}\end{array}$ \\
\hline MK & $0.91 \pm 0.03$ & $1.06 \pm 0.02$ & $t=-23.109$ & $<.001$ \\
\hline MD & $1.14 \pm 0.01$ & $0.80 \pm 0.01$ & $t=93.275$ & $<.001$ \\
\hline FA & $0.42 \pm 0.01$ & $0.66 \pm 0.02$ & $t=-41.311$ & $<.001$ \\
\hline$A D$ & $2.03 \pm 0.03$ & $2.27 \pm 0.02$ & $t=-35.271$ & $<.001$ \\
\hline $\mathrm{RD}$ & $0.75 \pm 0.02$ & $0.97 \pm 0.03$ & $t=-55.311$ & $<.001$ \\
\hline AK & $0.56 \pm 0.01$ & $0.86 \pm 0.01$ & $t=-44.834$ & $<.001$ \\
\hline RK & $0.67 \pm 0.03$ & $0.91 \pm 0.02$ & $t=-64.167$ & $<.001$ \\
\hline \multicolumn{5}{|c|}{$\begin{array}{l}\text { Note. }- \text { Abbreviation: } M K=\text { mean kurtosis (unitless); } M D=\text { mean diffusivity }\left(\times 10^{-3} \mathrm{~mm}^{2} / \mathrm{s}\right) ; \mathrm{FA}= \\
\text { fractional anisotropy (unitless); } \mathrm{AD}=\text { axial diffusivity }\left(\times 10^{-3} \mathrm{~mm}^{2} / \mathrm{s}\right) ; \mathrm{RD}=\text { radial diffusivity }\left(\times 10^{-3}\right. \\
\left.\mathrm{mm}^{2} / \mathrm{s}\right) ; \mathrm{AK}=\text { axial kurtosis (unitless); } \mathrm{RK}=\text { radial kurtosis (unitless). } \mathrm{DKI} \text { metrics from lesion for } \\
\text { patients with T2 hyperintense and from gray/white matter for patients with no T2 hyperintense. }\end{array}$} \\
\hline
\end{tabular}

\section{DKI metrics and therapeutic effect}

Tables 4 and 5 showed that DKI parameters of sections from lesions and the values in sections of normal-appearing GM/WM were compared before and after one course of treatment in MS patients with T2 hyperintense $(n=15)$ and MS patients without T2 hyperintense lesions $(n=33)$, respectively. After the end of the treatment, MK, FA, AD, RD, AK and RK values were increased while the MD value was decreased compared to that before the treatment; the difference was statistically significant (all $P<0.001$ ) in MS patients with T2 hyperintense, although no significant change was noted in the number, location, and signal intensity of T2-hyperintense lesions. In MS patients without T2 hyperintense, normal-appearing GM-MK and normal-appearing WM-FA and WM-AD increased significantly (all $P<0.001$ ), while normalappearing WM-MD decreased $(P=0.019)$ after treatment compared to that before treatment. 
Table 4

Comparison of DKI metrics before and after treatment in MS patients with T2 hyperintense $(n=15)$

\begin{tabular}{|c|c|c|c|c|}
\hline Metrics\$ & Before treatment & $\begin{array}{l}\text { After } \\
\text { treatment }\end{array}$ & Parameter & $P$ value $^{\ddagger}$ \\
\hline MK & $0.91 \pm 0.03$ & $0.98 \pm 0.05$ & $t=10.800$ & $<.001$ \\
\hline MD & $1.14 \pm 0.01$ & $1.12 \pm 0.01$ & $t=6.441$ & $<.001$ \\
\hline FA & $0.42 \pm 0.01$ & $0.45 \pm 0.02$ & $t=10.247$ & $<.001$ \\
\hline$A D$ & $2.03 \pm 0.03$ & $2.06 \pm 0.02$ & $t=11.156$ & $<.001$ \\
\hline $\mathrm{RD}$ & $0.75 \pm 0.02$ & $0.77 \pm 0.01$ & $t=5.566$ & $<.001$ \\
\hline AK & $0.56 \pm 0.01$ & $0.58 \pm 0.02$ & $t=7.122$ & $<.001$ \\
\hline RK & $0.67 \pm 0.03$ & $0.71 \pm 0.02$ & $t=12.564$ & $<.001$ \\
\hline \multicolumn{5}{|c|}{$\begin{array}{l}\text { Note. }- \text { Abbreviation: } M K=\text { mean kurtosis (unitless); } M D=\text { mean diffusivity }\left(\times 10^{-3} \mathrm{~mm}^{2} / \mathrm{s}\right) ; \mathrm{FA}= \\
\text { fractional anisotropy (unitless); } \mathrm{AD}=\text { axial diffusivity }\left(\times 10^{-3} \mathrm{~mm}^{2} / \mathrm{s}\right) ; \mathrm{RD}=\text { radial diffusivity }\left(\times 10^{-3}\right. \\
\left.\mathrm{mm}^{2} / \mathrm{s}\right) ; \mathrm{AK}=\text { axial kurtosis (unitless); } \mathrm{RK}=\text { radial kurtosis (unitless). } \mathrm{GM}=\text { gray matter; } \mathrm{WM}=\text { white } \\
\text { matter. DKI metrics from lesion for patients with } \mathrm{T} 2 \text { hyperintense. }\end{array}$} \\
\hline
\end{tabular}


Table 5

Comparison of DKI metrics before and after treatment in MS patients with no T2 hyperintense $(n=33)$

\begin{tabular}{|c|c|c|c|c|c|}
\hline Metrics§ & Location & Before treatment & $\begin{array}{l}\text { After } \\
\text { treatment }\end{array}$ & Parameter & $P$ value $^{\ddagger}$ \\
\hline \multirow[t]{2}{*}{ MK } & GM & $1.08 \pm 0.02$ & $1.09 \pm 0.04$ & $t=5.200$ & $<.001$ \\
\hline & WM & $1.12 \pm 0.13$ & $1.11 \pm 0.03$ & $t=1.153$ & .258 \\
\hline \multirow[t]{2}{*}{ MD } & GM & $0.71 \pm 0.01$ & $0.71 \pm 0.12$ & $t=1.153$ & .59 \\
\hline & WM & $0.90 \pm 0.02$ & $0.88 \pm 0.02$ & $t=2.141$ & .019 \\
\hline \multirow[t]{2}{*}{ FA } & GM & $0.80 \pm 0.12$ & $0.81 \pm 0.03$ & $t=1.391$ & .174 \\
\hline & WM & $0.52 \pm 0.04$ & $0.53 \pm 0.03$ & $t=4.983$ & $<.001$ \\
\hline \multirow[t]{2}{*}{$A D$} & GM & $1.54 \pm 0.02$ & $1.54 \pm 0.03$ & $t=1.168$ & .877 \\
\hline & WM & $1.67 \pm 0.03$ & $1.71 \pm 0.04$ & $t=6.145$ & $<.001$ \\
\hline \multirow[t]{2}{*}{$\mathrm{RD}$} & GM & $0.65 \pm 0.04$ & $0.64 \pm 0.02$ & $t=1.213$ & .644 \\
\hline & WM & $0.68 \pm 0.02$ & $0.67 \pm 0.03$ & $t=1.225$ & .678 \\
\hline \multirow[t]{2}{*}{ AK } & GM & $0.59 \pm 0.01$ & $0.59 \pm 0.02$ & $t=1.247$ & .358 \\
\hline & WM & $0.60 \pm 0.02$ & $0.60 \pm 0.04$ & $t=1.328$ & .558 \\
\hline \multirow[t]{2}{*}{ RK } & GM & $0.91 \pm 0.03$ & $0.90 \pm 0.02$ & $t=1.928$ & .093 \\
\hline & WM & $0.94 \pm 0.01$ & $0.93 \pm 0.02$ & $t=1.557$ & .107 \\
\hline \multicolumn{6}{|c|}{$\begin{array}{l}\text { Note. }- \text { Abbreviation: } M K=\text { mean kurtosis (unitless); } M D=\text { mean diffusivity }\left(\times 10^{-3} \mathrm{~mm}^{2} / \mathrm{s}\right) ; \mathrm{FA}= \\
\text { fractional anisotropy (unitless); } \mathrm{AD}=\text { axial diffusivity }\left(\times 10^{-3} \mathrm{~mm}^{2} / \mathrm{s}\right) ; \mathrm{RD}=\text { radial diffusivity }\left(\times 10^{-3}\right. \\
\left.\mathrm{mm}^{2} / \mathrm{s}\right) ; \mathrm{AK}=\text { axial kurtosis (unitless); } \mathrm{RK}=\text { radial kurtosis (unitless). } \mathrm{GM}=\text { gray matter; WM = white } \\
\text { matter. DKI metrics from } \mathrm{GM} \text { and WM for patients with no T2 hyperintense. } \\
\text { SData are } \pm \text { standard deviation. }\end{array}$} \\
\hline
\end{tabular}

\section{DKI metrics and disability as determined by the EDSS}

In the MS patients of CSC of T2-hyperintense lesion, a significantly converse correlation was established between the EDSS score and MK value before and after treatment $(r=-0.634, P=0.011$ and $r=-0.645, P=$ 0.009 , respectively). In addition, significant converse correlations were established between the EDSS score and MK value of normal-appearing GM before and after the treatment $(r=-0.630, P=0.011$ and $r=$ $-0.377, P=0.031$, respectively) (Fig. 3 ). No significant correlations were established between the EDSS score and other DKI parameters in MS patients. 


\section{Discussion}

CSC injuries detected on MRI have critical diagnostic and prognostic value for MS[23]. Routine MRI of CSC could detect lesions with only limited micropathological change. Ozturk showed that T2*-weighted gradient-echo scans in the spinal cord reveal MS lesions with greater regularity compared to routine T2weighted spin-echo imaging[24]. Thus, we used T2*-weighted imaging to count and outline all T2hyperintense lesions in MS patients, which is consistent with the sequence used by chuhutin et al[25].

In the current study, MS patients with normal-appearing GM and WM had damage likely due to CSC microscopic injuries that were not detected by routine MRI. The MS pathology of CSC included axonal loss, inflammation, demyelination, and gliosis; each of these features could be inferred with quantitative MR metrics of tissue structure[26-29]. Therefore, the micropathological changes of CSC for MS patients played a critical role in determining the therapeutic plans and evaluating prognosis. In line with previous DKI studies[12], our DKI metrics from T2-hyperintense lesions and normal-appearing GM and WM suggested extensive microscopic damage of CSC in MS patients compared to healthy subjects, suggesting that quantitative MR of CSC overcomes clinical-radiology paradox. Demyelination seems to play a part in the early phase, while other processes, such as axonal loss, are prominent in the later course of the disease[12]. Both axonal loss and demyelination decrease FA and AD, inflammation, and gliosis, increasing in $\mathrm{MD}[23,30]$.

Accumulating evidence indicated that CSC injury in MS patients is not only limited to WM but also involves the GM[31-34]. High annualized spinal cord atrophy rates were correlated with reductions in gray matter volume. The study by Kearney et al[17]. and Schlaeger et al[16]. suggested that loss of GM is the main contributor to progressive disability. The current study showed that only the MK and RK values of normal-appearing GM were significantly reduced in MS patients compared to the healthy participants, indicating that GM injury persists below the resolution of routine T2-weighted imaging, MK and AK could serve as a more sensitive potential biomarker for evaluating the GM than DTI-metrics in the early diagnosis of the disease. Subsequently, MK and AK may provide more information that is different and complementary to that obtained with DTI on the evaluation of GM damage. Decreased MK is likely associated with neuronal shrinkage, pronounced gliosis, and demyelination of the spinal cord GM[16]. This phenomenon was consistent with muscular atrophy (especially in the intrinsic hand muscles) for the majority of MS patients assessed at autopsy.

Additionally, we found severe lesions than in normal-appearing GM and WM for MS patients. Lukas et al. implied that patients suffering from disease progression during the follow-up period had significantly higher rates of CSC atrophy than patients with a stable disease course[7]. One of the main features of progressive patients with MS was the severe and extensive spinal cord involvement. A large number of lesions of CSC were observed in progressive MS than in relapsing MS phenotypes[37, 38]. In 15 MS patients with T2-hyperintense lesion, 14 were progressive MS, including 10 SPMS and 4 PPMS, confirming a greater number of lesions in patients with SPMS and PPMS vs. RRMS patients[39, 40]. This might be correlated with a greater axonal loss in this PPMS phenotype. 
In the initial MR studies, a converse association was found between MK from the lesions and normalappearing GM and the EDSS score in MS patients, implying that potential biomarkers of MK monitor the disability in MS. Also, MK might help to improve the monitoring of disease progression and prediction of disease evolution.

The variability of axon and dendrite orientations-termed neurite orientation dispersion-is reduced in the spinal cords of patients with MS[41]. This finding could provide a novel biomarker for MS prognosis and therapeutic efficacy. Axonal loss in the CSC is a key source of monitoring disease progression in patients with MS. Although no increase was detected in the number of lesions after the treatment in the current study. FA and $A D$ values originated from lesions and normal-appearing WM are significantly increased (all $P<0.001$ ), which might indicate regeneration of neural axons. Similarly, MK is increased (all $P<$ 0.001) at lesions and normal-appearing GM, indicating that MK may assess therapeutic efficacy[38].

\section{Limitations}

First, the number of MS patients was low and not selected according to MS phenotypes. Reportedly, FA and MD of CSC are differences among different subtypes of MS patients[38]. However, the differences in MK have not been reported among various subtypes of MS patients, necessitating in-depth research. Second, the FA and MD estimated inside DKI framework may be more accurate in Thaler's study, which is what we need to improve in the future[42]. Third, the resolution and SNR of the DKI acquisition are not sufficiently high to differentiate the gray/white matter, future research needs to use a high in-plane resolution of DWI acquisitions (e.g. $1 \mathrm{~mm}^{2}$ ). Fourth, transferring the ROls does not correct for the distortion from the EPI readout, low SNR and resolution in DKI images. Fifth, this cross-sectional study could not demonstrate how DKI-metrics dynamically change as MS advances continually. Future longitudinal studies are warranted to examine whether the current approach could be used to monitor the disease progression of MS. Sixth, our study was limited to the cervical spinal cord. Regina et al. demonstrated that remarkable GM atrophy is present at lower thoracic levels, which might affect the EDSS[43].

\section{Conclusion}

Spinal cord micropathological abnormalities at disease onset have critical implications for diagnosis and prognosis. Quantitative DKI metrics of CSC could be sensitive to the axonal loss and other pathological processes of lesions and normal-appearing GM and WM, assigning quantitative MRI a key role to elucidate the association between disability and spinal cord abnormalities in MS patients.

\section{Declarations}

\section{Acknowledgments}

The authors thank their patients and volunteers for participating in this study. 


\section{Authors' contributions}

BBY conceived and designed the study, LD supervised the research. BBY, SZQ and ZBX collected and analyzed data. ZXN, JYG interpreted data. LD substantively revised it. All authors read and approved the final manuscript.

\section{Funding}

This study was supported by the Natural Science Foundation of Jilin Province (Grant No.YDZJ202101ZYTS084 and YDZJ202101ZYTS019).

\section{Availability of data and materials}

The datasets collected and analyzed during the current study are available from the corresponding author upon reasonable request.

\section{Ethical Approval}

The study was approved by the Medical Research Ethics Committee and the Institutional Review Board of the First Hospital of Jilin University (2020-485) and was performed in accordance with the principles expressed in the Declaration of Helsinki. All study participants provided written informed consent.

\section{Consent for publication}

Not applicable.

\section{Competing interests}

The authors declare that the article content was composed in the absence of any commercial or fnancial relationships that could be construed as a potential confict of interest.

\section{References}

1. Reich DS, Lucchinetti CF, Calabresi PA. Multiple Sclerosis. N Engl J Med. 2018; 378(2):169-180.

2. Stenager E. A global perspective on the burden of multiple sclerosis. Lancet Neurol. 2019; 18(3):227228.

3. Ciccarelli O, Cohen JA, Reingold SC, Weinshenker BG. Spinal cord involvement in multiple sclerosis and neuromyelitis optica spectrum disorders. Lancet Neurol. 2019; 18(2):185-197.

4. Filippi M, Rocca MA. Novel MRI approaches to assess patients with multiple sclerosis. Curr Opin Neurol. 2010; 23(3):212-217.

5. Kearney H, Yiannakas MC, Samson RS, Wheeler-Kingshott CA, Ciccarelli O, Miller DH. Investigation of magnetization transfer ratio-derived pial and subpial abnormalities in the multiple sclerosis spinal cord. Brain. 2014; 137(Pt 9):2456-2468. 
6. Evangelou N, DeLuca GC, Owens T, Esiri MM. Pathological study of spinal cord atrophy in multiple sclerosis suggests limited role of local lesions. Brain. 2005; 128(Pt 1):29-34.

7. Lukas C, Knol DL, Sombekke MH, Bellenberg B, Hahn HK, Popescu V, et al. Cervical spinal cord volume loss is related to clinical disability progression in multiple sclerosis. J Neurol Neurosurg Psychiatry. 2015; 86(4):410-418.

8. Rocca MA, Horsfield MA, Sala S, Copetti M, Valsasina P, Mesaros S, et al. A multicenter assessment of cervical cord atrophy among MS clinical phenotypes. Neurology. 2011; 76(24):2096-2102.

9. Rocca MA, Valsasina P, Damjanovic D, Horsfield MA, Mesaros S, Stosic-Opincal T, et al. Voxel-wise mapping of cervical cord damage in multiple sclerosis patients with different clinical phenotypes. $J$ Neurol Neurosurg Psychiatry. 2013; 84(1):35-41.

10. Kearney H, Miller DH, Ciccarelli O. Spinal cord MRI in multiple sclerosis-diagnostic, prognostic and clinical value. Nat Rev Neurol. 2015; 11(6):327-338.

11. Oh J, Saidha S, Chen M, Smith SA, Prince J, Jones C, et al. Spinal cord quantitative MRI discriminates between disability levels in multiple sclerosis. Neurology. 2013; 80(6):540-547.

12. Raz E, Bester M, Sigmund EE, Tabesh A, Babb JS, Jaggi H, et al. A better characterization of spinal cord damage in multiple sclerosis: a diffusional kurtosis imaging study. AJNR Am J Neuroradiol. 2013; 34(9):1846-1852.

13. Gass A, Rocca MA, Agosta F, Ciccarelli O, Chard D, Valsasina P, et al. MRI monitoring of pathological changes in the spinal cord in patients with multiple sclerosis. Lancet Neurol. 2015; 14(4):443-454.

14. Zollinger LV, Kim TH, Hill K, Jeong EK, Rose JW. Using diffusion tensor imaging and immunofluorescent assay to evaluate the pathology of multiple sclerosis. J Magn Reson Imaging. 2011; 33(3):557-564.

15. Panara V, Navarra R, Mattei PA, Piccirilli E, Bartoletti V, Uncini A, et al. Correlations between cervical spinal cord magnetic resonance diffusion tensor and diffusion kurtosis imaging metrics and motor performance in patients with chronic ischemic brain lesions of the corticospinal tract. Neuroradiology. 2019; 61(2):175-182.

16. Schlaeger R, Papinutto N, Panara V, Bevan C, Lobach IV, Bucci M, et al. Spinal cord gray matter atrophy correlates with multiple sclerosis disability. Ann Neurol. 2014; 76(4):568-580.

17. Kearney H, Schneider T, Yiannakas MC, Altmann DR, Wheeler-Kingshott CA, Ciccarelli O, et al. Spinal cord grey matter abnormalities are associated with secondary progression and physical disability in multiple sclerosis. J Neurol Neurosurg Psychiatry. 2015; 86(6):608-614.

18. Jensen JH, Helpern JA, Ramani A, Lu H, Kaczynski K. Diffusional kurtosis imaging: the quantification of non-gaussian water diffusion by means of magnetic resonance imaging. Magn Reson Med. 2005; 53(6):1432-1440.

19. Zipp F, Oh J, Fragoso YD, Waubant E. Implementing the 2017 McDonald criteria for the diagnosis of multiple sclerosis. Nat Rev Neurol. 2019; 15(8):441-445.

20. By S, Xu J, Box BA, Bagnato FR, Smith SA. Application and evaluation of NODDI in the cervical spinal cord of multiple sclerosis patients. Neuroimage Clin. 2017; 15:333-342. 
21. Tabesh A, Jensen JH, Ardekani BA, Helpern JA. Estimation of tensors and tensor-derived measures in diffusional kurtosis imaging. Magn Reson Med. 2011; 65(3):823-836.

22. Hori M, Fukunaga I, Masutani Y, Nakanishi A, Shimoji K, Kamagata K, et al. New diffusion metrics for spondylotic myelopathy at an early clinical stage. Eur Radiol. 2012; 22(8):1797-1802.

23. Eden D, Gros C, Badji A, Dupont SM, De Leener B, Maranzano J, et al. Spatial distribution of multiple sclerosis lesions in the cervical spinal cord. Brain. 2019; 142(3):633-646.

24. Ozturk A, Aygun N, Smith SA, Caffo B, Calabresi PA, Reich DS. Axial 3D gradient-echo imaging for improved multiple sclerosis lesion detection in the cervical spinal cord at 3T. Neuroradiology. 2013; 55(4):431-439.

25. Chuhutin A, Hansen B, Wlodarczyk A, Owens T, Shemesh N, Jespersen SN. Diffusion Kurtosis Imaging maps neural damage in the EAE model of multiple sclerosis. Neuroimage. 2020; 208:116406.

26. Budde MD, Kim JH, Liang HF, Russell JH, Cross AH, Song SK. Axonal injury detected by in vivo diffusion tensor imaging correlates with neurological disability in a mouse model of multiple sclerosis. Nmr Biomed. 2008; 21(6):589-597.

27. Oh J, Zackowski K, Chen M, Newsome S, Saidha S, Smith SA, et al. Multiparametric MRI correlates of sensorimotor function in the spinal cord in multiple sclerosis. Mult Scler. 2013; 19(4):427-435.

28. Valsasina P, Rocca MA, Agosta F, Benedetti B, Horsfield MA, Gallo A, et al. Mean diffusivity and fractional anisotropy histogram analysis of the cervical cord in MS patients. Neuroimage. 2005; 26(3):822-828.

29. Ciccarelli O, Wheeler-Kingshott CA, McLean MA, Cercignani M, Wimpey K, Miller DH, et al. Spinal cord spectroscopy and diffusion-based tractography to assess acute disability in multiple sclerosis. Brain. 2007; 130(Pt 8):2220-2231.

30. Schmierer K, Wheeler-Kingshott CA, Boulby PA, Scaravilli F, Altmann DR, Barker GJ, et al. Diffusion tensor imaging of post mortem multiple sclerosis brain. Neuroimage. 2007; 35(2):467-477.

31. Farrell JA, Smith SA, Gordon-Lipkin EM, Reich DS, Calabresi PA, van Zijl PC. High b-value q-space diffusion-weighted MRI of the human cervical spinal cord in vivo: feasibility and application to multiple sclerosis. Magn Reson Med. 2008; 59(5):1079-1089.

32. Bot JC, Blezer EL, Kamphorst W, Lycklama ANG, Ader HJ, Castelijns JA, et al. The spinal cord in multiple sclerosis: relationship of high-spatial-resolution quantitative MR imaging findings to histopathologic results. Radiology. 2004; 233(2):531-540.

33. Gilmore CP, Bo L, Owens T, Lowe J, Esiri MM, Evangelou N. Spinal cord gray matter demyelination in multiple sclerosis-a novel pattern of residual plaque morphology. Brain Pathol. 2006; 16(3):202-208.

34. Gilmore CP, Geurts JJ, Evangelou N, Bot JC, van Schijndel RA, Pouwels PJ, et al. Spinal cord grey matter lesions in multiple sclerosis detected by post-mortem high field MR imaging. Mult Scler. 2009; 15(2):180-188.

35. Gilmore CP, Donaldson I, Bo L, Owens T, Lowe J, Evangelou N. Regional variations in the extent and pattern of grey matter demyelination in multiple sclerosis: a comparison between the cerebral cortex, 
cerebellar cortex, deep grey matter nuclei and the spinal cord. J Neurol Neurosurg Psychiatry. 2009; 80(2):182-187.

36. Bot JC, Barkhof F, Polman CH, Lycklama ANG, de Groot V, Bergers E, et al. Spinal cord abnormalities in recently diagnosed MS patients: added value of spinal MRI examination. Neurology. 2004; 62(2):226-233.

37. Valsasina P, Aboulwafa M, Preziosa P, Messina R, Falini A, Comi G, et al. Cervical Cord T1-weighted Hypointense Lesions at MR Imaging in Multiple Sclerosis: Relationship to Cord Atrophy and Disability. Radiology. 2018; 288(1):234-244.

38. Lycklama G, Thompson A, Filippi M, Miller D, Polman C, Fazekas F, et al. Spinal-cord MRI in multiple sclerosis. Lancet Neurol. 2003; 2(9):555-562.

39. Weier K, Mazraeh J, Naegelin Y, Thoeni A, Hirsch JG, Fabbro T, et al. Biplanar MRI for the assessment of the spinal cord in multiple sclerosis. Mult Scler. 2012; 18(11):1560-1569.

40. Grussu F, Schneider T, Tur C, Yates RL, Tachrount M, lanus A, et al. Neurite dispersion: a new marker of multiple sclerosis spinal cord pathology? Ann Clin TransI Neurol. 2017; 4(9):663-679.

41. Klawiter EC, Schmidt RE, Trinkaus K, Liang HF, Budde MD, Naismith RT, et al. Radial diffusivity predicts demyelination in ex vivo multiple sclerosis spinal cords. Neuroimage. 2011; 55(4):14541460.

42. Thaler C, Kyselyova AA, Faizy TD, Nawka MT, Jespersen S, Hansen B, et al. Heterogeneity of multiple sclerosis lesions in fast diffusional kurtosis imaging. Plos One. 2021; 16(2):e245844.

43. Schlaeger R, Papinutto N, Zhu AH, Lobach IV, Bevan CJ, Bucci M, et al. Association Between Thoracic Spinal Cord Gray Matter Atrophy and Disability in Multiple Sclerosis. Jama Neurol. 2015; 72(8):897904.

\section{Figures}




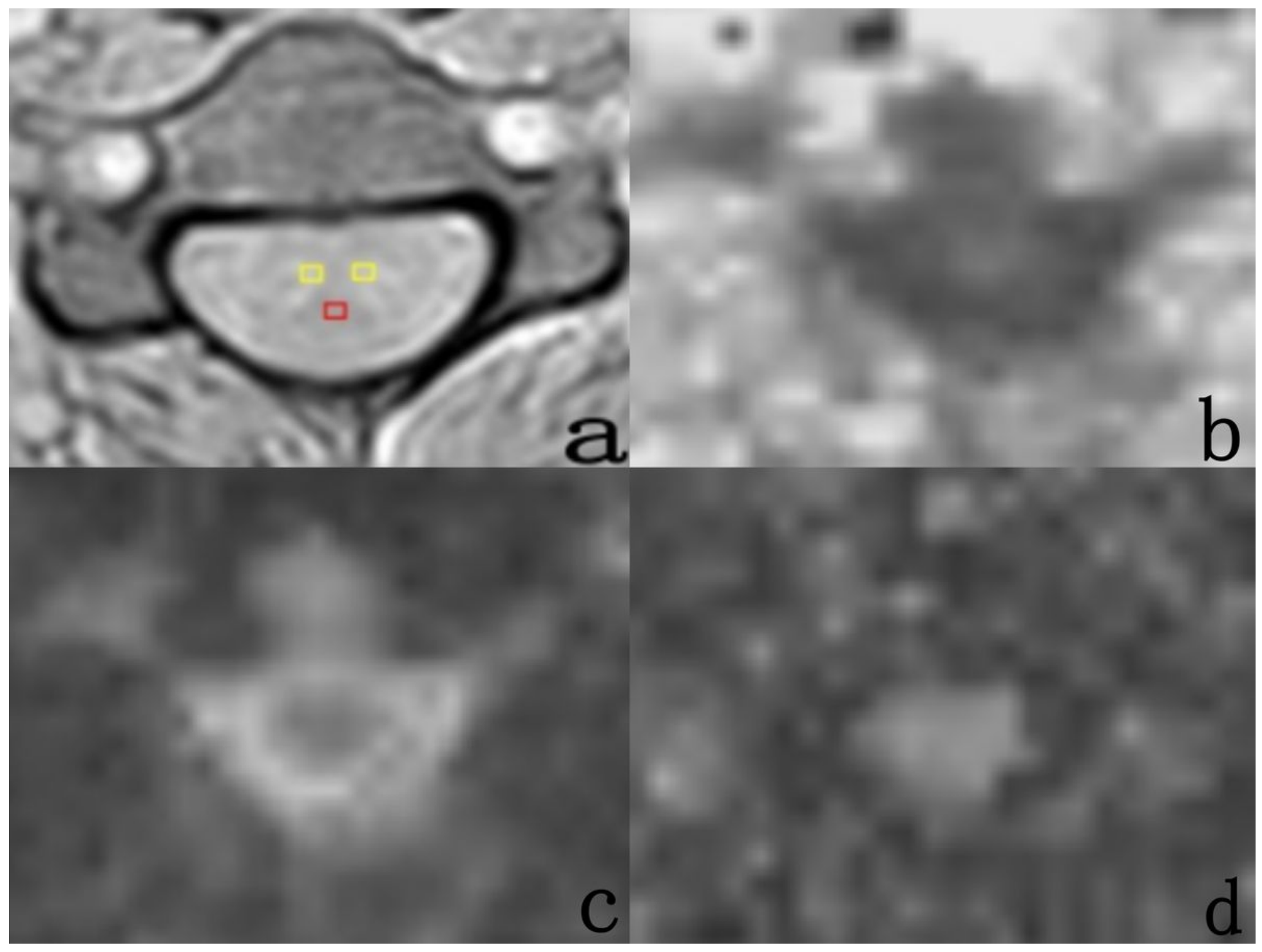

\section{Figure 1}

Axial T2* weighted image (a) of the cervical spinal cord at C2/C3 disk level shows the location of ROls placed in the gray matter of the bilateral ventral horns (yellow) and white matter of the dorsal column (red). Corresponding mean kurtosis (b), mean diffusivity (c), and fractional anisotropy (d) maps. Images are from a healthy subject. 

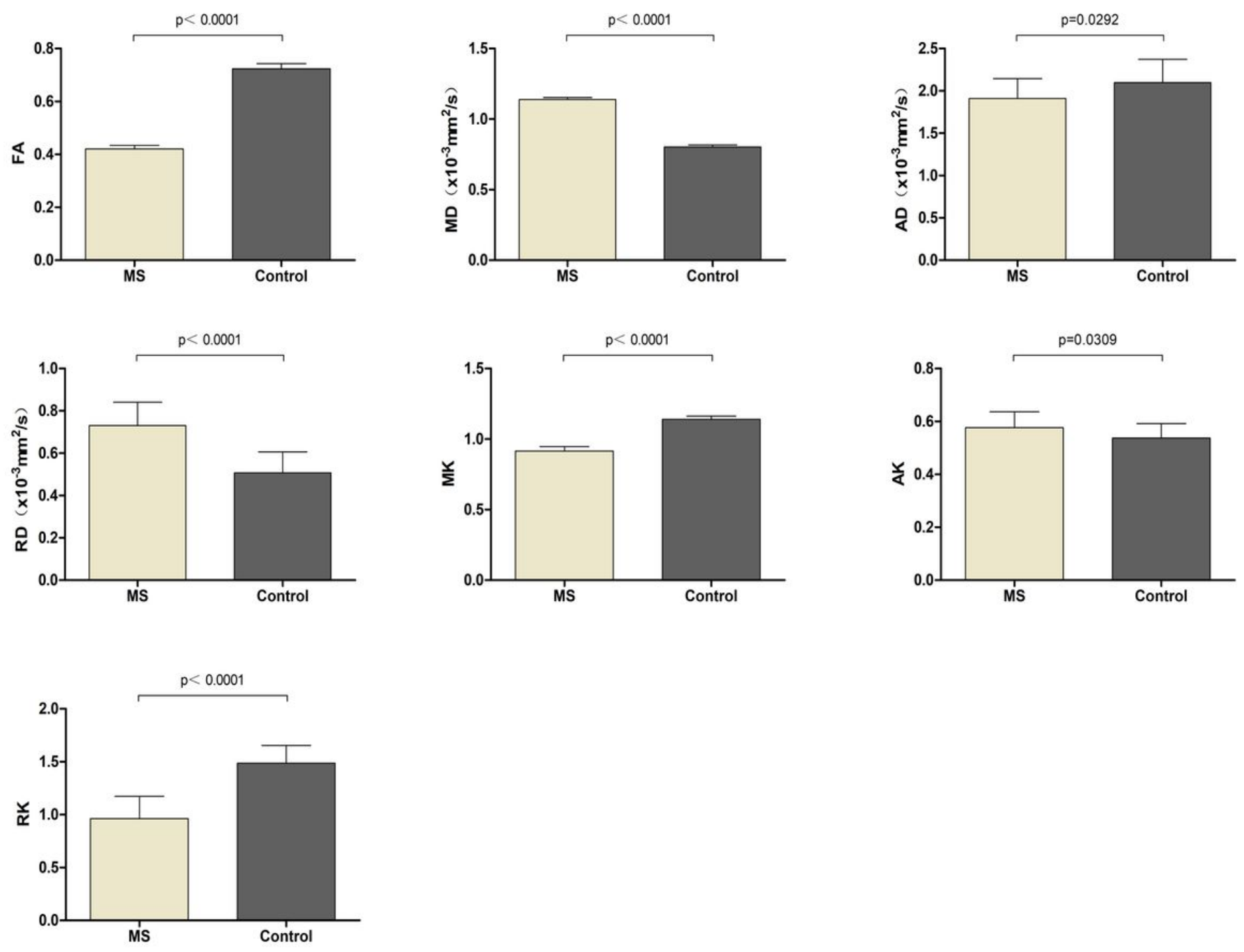

\section{Figure 2}

The box-and-whisker plots shows the distribution of $F A, M D, A D, R D, M K, A K$ and RK value in cervical spinal cord of MS patients and healthy subjects. Patients with MS had a significantly smaller FA, AD, MK, and RK value as well as higher MD, RD and AK value than healthy subjects (all $P$.005). 

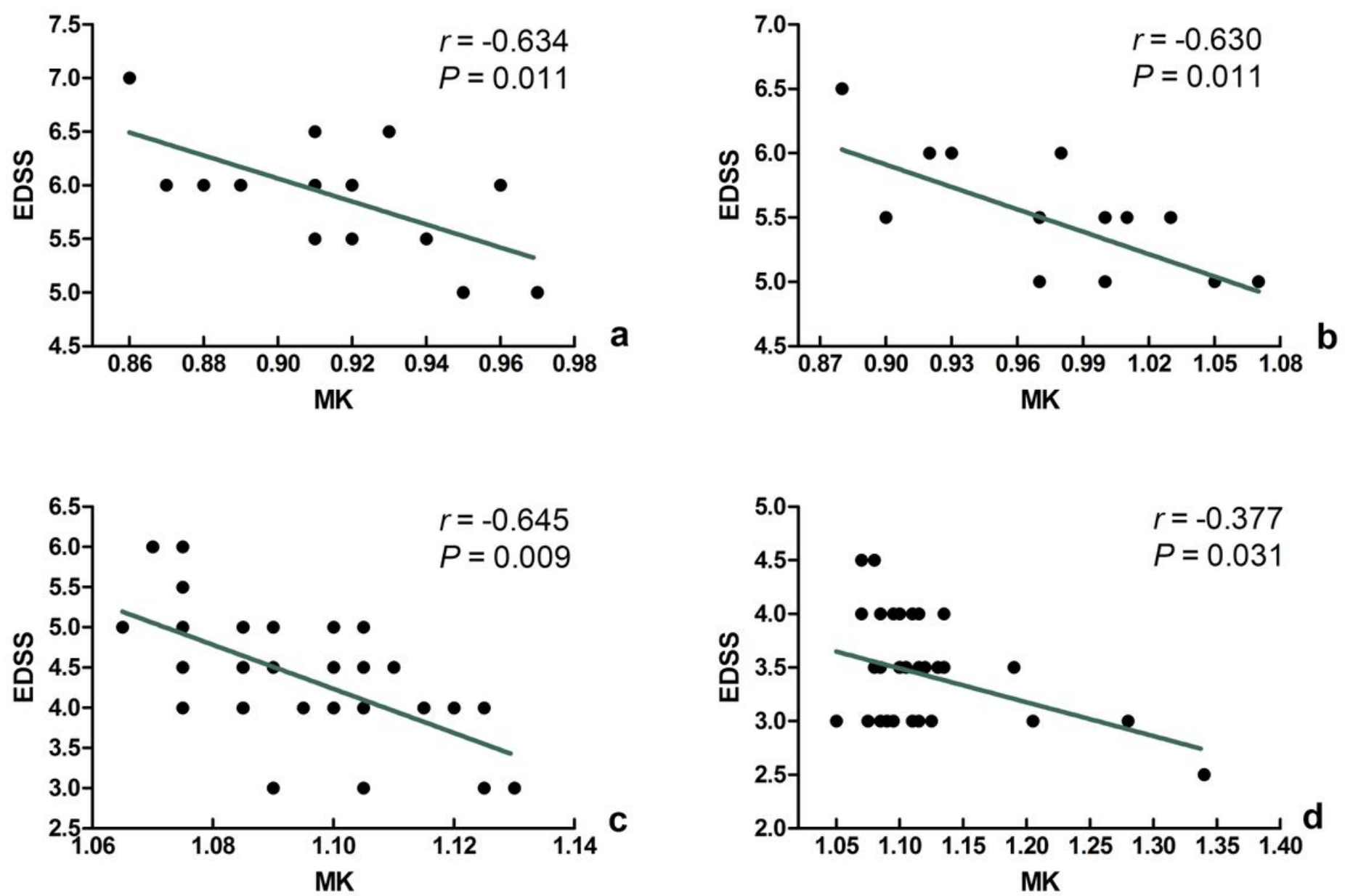

Figure 3

Scatterplots of the relationship between EDSS score and MK. a. shows that the MK value of patients with T2-hyperintense lesions is negatively correlated with the EDSS score; $\mathbf{b}$. shows that the MK value of patients with T2-hyperintense lesions is negatively correlated with the EDSS score after treatment; c.shows that the MK value of patients without T2-hyperintense lesions is negatively correlated with the EDSS score; $\mathbf{d}$. shows that the MK value of patients without T2-hyperintense lesions is negatively correlated with the EDSS score after treatment. 